\title{
The New Thermo-magnetic Effect in Metals
}

\author{
B.V.Vasiliev \\ Independent Researcher, Russia \\ *Corresponding Author: bv.vasiliev@narod.ru
}

Copyright (C)2014 Horizon Research Publishing All rights reserved.

\begin{abstract}
The magnetic field which induced by the thermo-electric current in metals was detected and measured using of a flux-gate magnetometer. It is shown that the application of a temperature gradient on a metal rod gives rise to a circulating current therein and induces a magnetic field in the vicinity of its surface. If a temperature gradient on a metal rod exists, the "hot" electrons flow from the heated region of a metal into a colder region and extrude "cold" electrons that form a current in opposite direction. Since the oppositely directed currents repel each other due to the interaction of magnetic fields, a convective loop of electron current formes inside a metalic sample. The magnetic field of this convection is directly proportional to the temperature gradient, the metal conductivity and inversely proportional to the temperature squared.
\end{abstract}

Keywords Metal, Magnetic Field, Thermoelectric Effect, Flux-gate Magnitometer

However, the thermopower is not a very valuable probe of fundamental electronic properties of a metal.

N.Ashcroft, N.Mermim: Solid State Physics, v.1, p.258. Holt, Reinhart and Winston,(1976)

\section{Introduction}

The thermoelectric phenomena which occur when gradient of temperature applied on a conducting specimen together with an external magnetic field is usually accepted to call as thermomagnetic effects.

There are a number of such phenomena which are well studied, for example, it is the Nernst-Ettingshausen effect or the Righi-Leduc effect. A number of these effects are discussed in detail in the monograph [1]. It is important to stress that all these phenomena are exist in semiconductors only.
The effect described below has a different nature. In fact we will discuss magnetic fields which are directly induced by gradient of temperature in metal specimens. Exactly, one can say, that we will consider magnetic fields of thermoelectric currents in metals.

Thermoelectric effects in metals are well studied [2], [3]. Originally the process of the heat transfer in electron gas of metals was described by Drude [2]. Late the Drude's model has been enhanced by Lorentz and Sommerfeld.

However, the magnetic field produced by a thermoelectric current of conduction electrons apparently has never been observed and investigated. Probably, the reason for this is that the courses related to this issue contain a some misunderstanding. The high thermal conductivity of metals is result of the ability of free electrons to transport a heat. At this process, the "hot" electrons flow from the heated region of a metal into a colder region and extrude "cold" electrons that form a current in opposite direction. Until now it has been commonly thought that in the case of a homogeneous metal, both these opposite electron current have a diffusive character and flow uniformly throughout the cross section of the sample. A deviation from uniformity of diffusive flux can be due to inhomogeneities in the sample only. However, this consideration has a mistake. Electric currents repel each other due to their magnetic interaction, if they flow in opposite directions. As a result, a peculiar electron convection occurs inside a metalic sample (see Fig.1), which leads to the existence of the magnetic field in the vicinity of the sample. The researching of this field is a focus of this work .

Experimentally this magnetic field is quite large and it can be detected by conventional flux-gate magnetometer. Therefore, when measuring small magnetic effects in metals with using SQUID-magnetometers, experimenters must to keep in mind that the specifying thermomagnetic effect can influence under certain conditions on the measurement results.

\section{Thermoelectric currents in a metal.}

The system of conducting electrons in a metal has the total density:

$$
n_{e}=z n_{i}
$$


where $z$ is the number of conducting electrons on a metal ion (the valence),

$n_{i}$ is the ion density in a metal.

As it was noted by A.Sommerfeld, all conducting electrons of a metal are divided into two groups.

In equilibrium state at temperature $\mathrm{T}$, there are nondegenerated electrons with density $n_{a}$, which are activated by a heat. They occupy levels above the Fermi level in the energy distribution.

The second group is formed by degenerate conducting electrons that occupy the energy levels below the Fermi level and have a density:

$$
n_{c}=n_{e}-n_{a} .
$$

Thermally activated non-degenerate electrons have the density:

$$
n_{a} \approx n_{e} \frac{T}{T_{F}} .
$$

They have the ability to descend from one energy level to another, and so they can transfer the heat at their moving in a metal.

Due to the fact that we consider a metal at room temperature $\left(T \ll T_{F}\right)$, the group of degenerate electrons includes almost all conducting electrons $n_{c} \cong n_{e}$. These electrons can not be involved in a process of a heat transfer. But, if the electric field exists inside the metal, they create a current. And they determine the electrical conductivity of a metal.

If the temperature gradient $\nabla T$ is attached to a metal rod, it will activate a thermal current of electrons from a hot region, where the electrons have higher speed and higher pressure.

Thermally activated electron betrays a transferring heat to the lattice due to inelastic collisions with phonons. Therefore, the free path of hot electrons between two successive collisions with phonons is inversely proportional to the phonon density $n_{p h}$ :

$$
\frac{1}{l_{p h}} \approx n_{p h} \cdot S
$$

Where $S$ is the electron-phonon scattering cross section. Due to the fact that we consider the metal at room temperature, and given the fact that the Debye temperature in most metals is of the same order of value, we can assume that

$$
S \approx n_{i}^{-2 / 3}
$$

The energy of the phonon gas in the Debye approximation [3]

$$
\mathcal{E}_{p h}=\frac{3 \pi^{4}}{5} n_{i} k T_{D}\left(\frac{T}{T_{D}}\right)^{4},
$$

where $T_{D}$ is the Debye temperature.

The phonon energy is approximately equal to $k T$, so their density can be described by:

$$
n_{p h} \approx \frac{3 \pi^{4}}{5} n_{i}\left(\frac{T}{T_{D}}\right)^{3},
$$

Subject to Eq.(5) phonons limit the mean free path of "hot" electrons by the value:

$$
l_{p h} \approx \frac{5}{3 \pi^{4}} n_{i}^{-1 / 3} \cdot\left(\frac{T_{D}}{T}\right)^{3}
$$

As the velocity of conducting electron is approximately equal to the Fermi velocity, the free path of electrons between two successive collisions with phonons is:

$$
\tau_{p h}=\frac{l_{p h}}{v_{F}} \approx \frac{\frac{5}{3 \pi^{4}}}{n_{i}^{1 / 3} v_{F}} \cdot\left(\frac{T_{D}}{T}\right)^{3} .
$$

To calculate the average velocity of the electrons we assume that two non-degenerate electrons coming from hot end and cold ends of a rod are thermalized in the point $x$ inside this metal rod. If the difference in their velocities are small, their average speed in the onedimensional model can be written as:

$$
\begin{array}{r}
v_{+}=\frac{1}{2}\left[v\left(x-v \tau_{p h}\right)-v\left(x+v \tau_{p h}\right)\right] \\
=-\tau_{p h} v \frac{d v}{d x}=-\tau_{p h} \frac{d}{d x}\left(\frac{v^{2}}{2}\right) .
\end{array}
$$

Turning to the case of three dimensions, we can write $[2]:$

$$
\mathbf{v}_{+}=-\frac{\tau_{e}}{6} \frac{d v^{2}}{d T} \nabla T=-\frac{\tau_{e}}{6} \frac{c_{v}}{m_{e} n_{e}} \nabla T
$$

where

$$
c_{v}=\frac{\pi^{2}}{3} k n_{e} \frac{k T}{\mathcal{E}_{F}}
$$

is the Sommerfeld's heat capacity of electron gas,

$m_{e}$ is the electron mass.

Given that electrons are passed to the point of the energy exchange from the distances which are equal to the mean free path, the velocity of thermal diffusion averaged over the whole electron gas becomes:

$$
<\mathbf{v}_{+}>=-\tau_{p h} \frac{\pi^{2}}{18} \frac{k}{m_{e}} \frac{T}{T_{F}} \nabla T .
$$

The considered heat flow induces the electron current

$$
\mathbf{j}_{+}=e n_{e}<\mathbf{v}_{+}>\approx \tau_{p h} \frac{\pi^{2}}{18} \frac{k e}{m_{e}} n_{e}\left(\frac{T}{T_{F}}\right) \nabla T
$$

and creates inside the metal rod the Seebeck's electric field:

$$
\mathbf{E}_{S}=Q_{S} \nabla T,
$$

under the action of which there occurs a reverse flow of electrons:

$$
\mathbf{j}_{-}=e n_{e} v_{-}=\sigma Q_{S} \nabla T
$$

where $\sigma$ is the conductivity of a metal.

The average velocity of electrons in this current [2]:

$$
<\mathbf{v}_{-}>=-\tau_{e} \frac{e}{m_{e}} \mathbf{E}_{S}
$$

where $\tau_{e}$ is the free time of electrons moving in an electric field.

The temperature dependence of this time is calculated in a number of courses on the theory of metals (see eg [5]) and it is approximately described by the equation:

$$
\tau_{e} \approx \frac{\hbar}{k T} \text {. }
$$

Hence we obtain

$$
\mathbf{j}_{-} \approx n_{e} \frac{\hbar}{k T} \frac{e^{2}}{m_{e}} Q_{S} \nabla T .
$$

and taking into account that $\mathbf{j}_{+}+\mathbf{j}_{-}=0$, we obtain the expression for the Seebeck coefficient:

$$
Q_{S} \simeq-\frac{\pi^{2}}{18} \frac{k}{e} \frac{T_{D}^{3}}{T T_{F}^{2}} .
$$




\section{The testing by means of the Wiedemann-Franz law.}

The Wiedemann-Franz law establishes the relationship between the electrical conductivity of the metal and its thermal conductivity. The compliance of obtained estimations with this law should indicate on their correctness. Let us check this criterion.

The thermal conductivity of gas is determined by the heat capacity $C$ of the environment that takes the heat from the hot gas particles, the gas particle velocity $v$ and the length of its free path $l[3]$ :

$$
\kappa=\frac{1}{3} C v l \approx \frac{1}{3} C v^{2} \tau .
$$

In the case of atomic or molecular gas the thermal conductivity is determined by their specific heat. The case of an electron gas is more complicated. The electronelectron interaction is weak in metals and the main mechanism of heat transfer is the electron-phonon interaction. The "hot" electrons transfer their energy to phonons. The phonon gas is the medium that takes the energy from the electrons. So in this case the specific heat of the phonon gas determines the electron gas thermal conductivity, which according to Eq.(6) approximately described by the equation:

$$
C_{p h} \approx \frac{12 \pi^{4}}{5} n_{i}\left(\frac{T}{T_{D}}\right)^{3},
$$

In view of the mean free time of non-degenerate electrons Eq.(9), we get:

$$
\kappa=\frac{2}{3} k n_{i}^{2 / 3} v_{F}
$$

Taking into account Eq.(18), we have:

$$
\sigma T=\frac{e^{2} n_{e}}{m_{e}} \frac{\hbar}{k}
$$

and thus we obtain the ratio:

$$
\frac{\kappa}{\sigma T} \approx 4 \frac{k^{2}}{e^{2}}
$$

which is in good agreement with the Wiedemann-Franz law.

\section{The electron convection in metals.}

Therefore, the gradient of the heat in the metallic rod with the conductivity $\sigma$ induces the current density:

$$
\mathbf{j}_{-}=-\mathbf{j}_{+}=\sigma \mathbf{E}_{S} \simeq \sigma \frac{T_{D}^{3}}{T T_{F}^{2}} \frac{k}{e} \nabla T .
$$

The magnetic interaction repels the electric currents if they flow in opposite directions. Therefore, in the case of a cylindrical specimen, the ends of which have different temperatures, currents $j_{+}$and $j_{-}$must flow through the diametrically opposite sides of cylinder (Fig.1). ${ }^{1}$

\footnotetext{
${ }^{1}$ This distribution of currents inside the metal body in its form is similar to the convective flow in the gas. It allows to call this phenomenon as "convection" for the sake of brevity, although the physics of these phenomena are quite different of course.
}

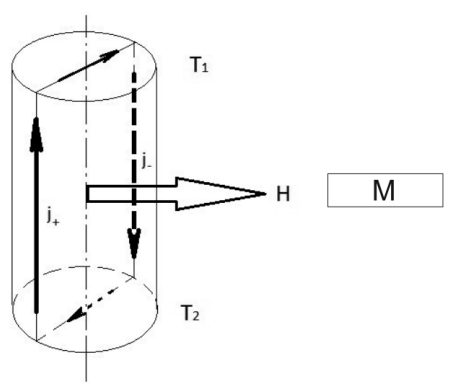

Figure 1. Currents induced by the temperature gradient in cylindrical metallic sample; $\mathrm{M}$ is the magnetometer.

Currents which was created by the gradient of the heat induce near the outer surface of the cylinder the magnetic field with intensity:

$$
H \approx \frac{2 j \cdot \pi R^{2}}{c R} \approx \Theta_{R}\left(\frac{\nabla T}{T^{2}}\right),
$$

where the constant

$$
\Theta_{R} \approx \frac{\pi^{3}}{9} \frac{k}{c e}(\sigma T) \frac{T_{D}^{3}}{T_{F}^{2}} R,
$$

where $R$ is the radius of cylinder.

\section{The measurement results}

When the temperature gradient of about $1 \frac{\mathrm{grad}}{\mathrm{cm}}$, which is not too difficult to create on a copper sample at room temperature, the induced magnetic field near the sample surface may reach $10^{-3} \mathrm{Oe}$, and it is not necessary to use a highly sensitive SQUID-magnetometer for its observation with which this effect was first observed by us.

The fluxgate-magnetometer is more simple, is more suitable for measurements in room temperature range and has quite enough sensitivity.

In our experiments [4], the fluxgate-magnetometer was placed near the middle of the metallic cylinder height. The temperature gradient was applied to cylinder. The temperatures of its ends was automatically measured at frequent intervals to determine the temperature gradient and the average temperature of the cylinder. The cylinder can rotate around its axis and fixed in a selected position for the registration component of the magnetic field perpendicular to the cylinder axis (see Fig.1).

\subsection{The determining of the "convective loop of current" orientation}

The first step in the investigation of the phenomenon of convection in the electron gas was the determination of the "loop of current" orientation inside the cylindrical sample. To do this, the cylinder has consistently turned a small angle (approximately equal to $15^{\circ}$ ) and measuring the projection of the induced field on the axis magnetometer was made. The measurement results are shown in Fig. 2. As it can be seen, the angular dependence of the induced field has the sinusoidal character. The shift in the angle for different samples in this figure due to the fact that in each case, the measurement started with an arbitrary angular orientation of the 


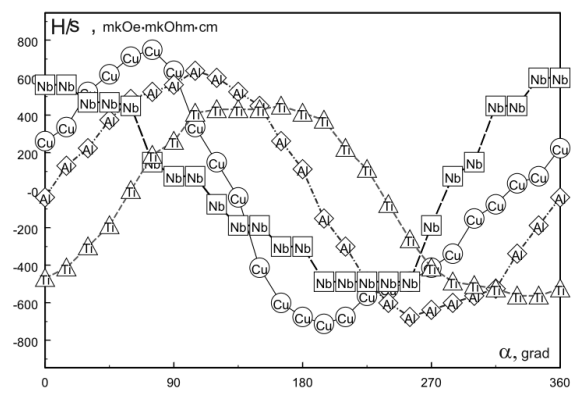

Figure 2. The angular dependence of the induced "convective" magnetic field, referred to the conductivity of the metal. The shift in the angle for different samples in this figure due to the fact that in each case, the measurement started with an arbitrary angular orientation of the cylinder. The angle of turnabout of the sample is shown on the horizontal axis. The induced magnetic field, referred to the conductivity of the metal, is shown on the ordinate.

cylinder.

After these measurements, the marks were deposited on the surface of the cylinder to denote the "loop of current" orientation.

Subsequent measurements were made at the particular orientation of the "loop of current" , at which the maximum of the field was obtained.

It should be emphasized that the position of "the convectional loop of current" inside the cylinder during the entire measurement period remained unchanged.

Special efforts to change the orientation of the "loop of current" have been made:

a) the significant hardening at the end face of the copper cylinder was made with a hammer,

b) a step at the end face of the cylinder was manufactured on the milling machine,

c) the copper cylinder was first heated to about $500^{\circ}$ $\mathrm{C}$ and then cooled either slowly or by quenching in water.

There was no any visible effect of these procedures on the "loop of current" orientation and the reason why "the convectional loop of current" has a continuing orientation in the cylinder remained unclear.

It should be noted, that in one of the cooper cylinder, "the convectional loop of current" was not originally found. But the normal effect appeared after a few light taps with a hammer on the end face of the cylinder.

Perhaps the fixing the convective flux of electrons in cylindrical samples has a purely geometric reason. All our samples were prepared on a lathe and they could be considered as circular in cross section in rough approximation. Thus all samples must be to have a certain ellipticity in their cross section. A repelling of convective electric currents each other makes energetically favorable a maximum distance between them. This ellipticity sectional samples could cause a rigid fixation of current paths. However this assumption has not been verified experimentally.
Table 1. The thermo-magnetic effect in long cylinders with $R=$ $1.5 \mathrm{~cm}$ at $T \approx 300 K$.

\begin{tabular}{||c|c|c|c|c|c||}
\hline \hline metal & $T_{F}, 10^{4} \mathrm{~K}$ & $T_{D}, \mathrm{~K}$ & $\begin{array}{c}\Theta_{\text {calc }} \\
\text { Eq. }(28)\end{array}$ & $\Theta_{\text {meas }}=\frac{H \cdot T^{2}}{\nabla T}$ & $\frac{\Theta_{\text {calc }}}{\Theta_{\text {meas }}}$ \\
\hline $\mathrm{Cu}$ & 8.1 & 343 & 48 & 43.5 & 1.1 \\
$\mathrm{Al}$ & 13.4 & 428 & 21 & 9.8 & 2.1 \\
$\mathrm{Ti}$ & 12.9 & 420 & 3.6 & 1.89 & 1.9 \\
$\mathrm{Nb}$ & 9.7 & 275 & 2.0 & 1.38 & 1.5 \\
\hline \hline
\end{tabular}

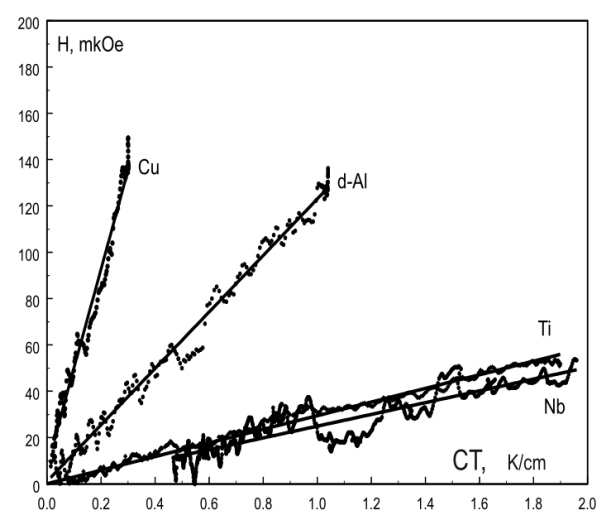

Figure 3. The dependence of the thermo-magnetic effect from the applied temperature gradient. On the abscissa the applied temperature gradient is shown in degrees per centimeter. On ordinate the induced magnetic field is plotted in the micro-gauss. The direct line carried out by least squares method.

\subsection{The "convection" of electron gas in different metals}

The thermo-magnetic effect was measured on four cylinders with the diameter of $30 \mathrm{~mm}$ and the length of about $150 \mathrm{~mm}$ from copper, duralumin, niobium and titanium. The linear dependence of the "convective" magnetic field from the applied temperature gradient is observed for all these metals (Fig. 3).

These measurements showed that the above estimate of the magnetic field induced by the heat flux is quite satisfactory agreement with measured data (see Table(1)).

\subsection{The temperature dependence of in- duced magnetic field}

The inverse quadratic dependence of the "convective" magnetic field which was predicted by Eq.(27) was tested on the copper sample. As it can be seen from Fig.4, the agreement of the observed data with the theoretical estimation can be considered satisfactory.

\section{Conclusion}

The above described thermo-magnetic effect is significant for good conductors. It can interfere with the study of other phenomena, where small magnetic effects in conductors are measured at the presence of temperature gradients. It can be assumed that this effect was observed previously in a number of measurements made with the help of high sensitive SQUID-magnetometer.

For example, in [6], we measured the small magnetic field resulting from rotation of the metal sample and it 


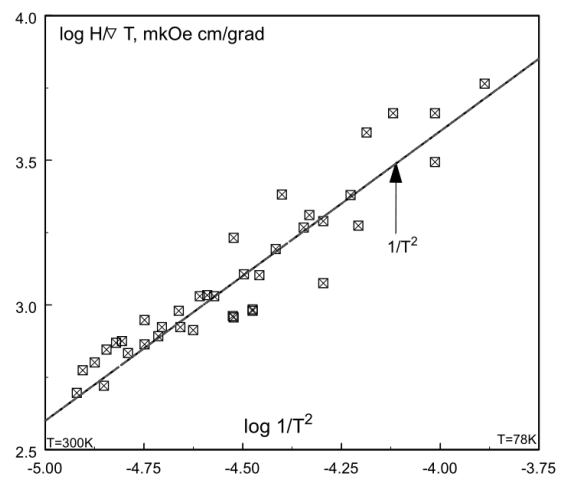

Figure 4. The temperature dependence of the "convective" magnetic field (referred to the temperature gradient) induced by the copper cylinder. On abscissa the logarithm of $\frac{1}{T^{2}}$ is plotted. On ordinate the logarithm of the magnetic field per temperature gradient in the $\mathrm{mkOe} \cdot \mathrm{cm} / \mathrm{grad}$. The line shows the dependence $T^{-2}$.

was proportional to its speed of rotation. It was supposed that this small field could be an effect of the inertial forces of rotation. However, it might seem that it was the result of excessive friction in one of the cryogenic bearings. The friction in one of bearing could create a temperature gradient on the metal sample and could induce "the loop of current" .

In [7], SQUID-magnetometer measured the magnetic field which was produced by a twisting of the metallic crystals to which the temperature gradient was applied. It was evident from the frequent conversation with the author of this work that it can not be excluded that at torsion there was a small bending of the sample. This bending was altering the projection of "the loop of current" on the axis of the SQUID-magnetometer, but this effect was interpreted as the field of twisting.

As for the reasons for fixing of the "convective loop of current" inside a cylindrical sample, it must be marked that the most simple "geometric" reason of it remains unexplored. The "convective loop of current" is formed because the "hot" and "cool" currents repel each other due to their magnetic interaction. Cylindrical specimens tested in the experiments described above, were made on lathes. Because of this, our samples can have a some small ellipticity. Due to the presence of such ellipticity, it would be energetically favorable for currents to pave their paths along lines through the ends of the major axis of the ellipse. It seems that this assumption puts everything in its place, also it has not been tested in the experiments described above.

\section{Acknowledgements}

The author is grateful for A.P.Gavrish's help with the measurements.

\section{REFERENCES}

[1] Callen H.B., Thermodynamics, Wiley, NY,Ch.17(1960).

[2] Ashcroft N.W., Mermin N.D.: Solid state physics, v 2., Holt,Rinehart and Winston, (1976)

[3] Kittel Ch.: Introduction to Solid State Physics, John Wiley@Son, NY, 1968.

[4] Vasiliev B.V., Gavrish A.P.: Preprint of Institute for Physical and Technical Problems, 96-1-3, Dubna (1996), (In Russian)

[5] Landau L.D., Lifshitz E.M.: Physical Kinetics, §80, Pergamon (1981)

[6] Vasiliev B.V.: JETP Letters, v.60, pp. 47-50, (1994).

[7] Zavaritsky N.V.: JETP Letters, v.16, pp.99-102 (1972). 